\title{
The Impact of Law Tech on the Future of Lawyers
}

Gabriela Bar <gabriela.bar@szostek-bar.pl>

WROCEAW, Poland

Silvia A. Carretta <silvia.carretta@ipandtechlab.com>

STOCKHOLM, Sweden

Shobana Iyer<shobana.iyer@swanchambers.com>

LONDON, England

\section{Abstract}

The future belongs to rapidly evolving technologies. The most fascinating and capable of revolutionising legal industry is likely to be with the use of Artificial Intelligence (AI). It will be the main driver of changes in the legal profession. In the digital world of the future, human lawyers will need to demonstrate emotional intelligence and a deep understanding of how technology can help them to provide better services. These qualities and skills will undoubtedly be just as valuable as formal legal knowledge. The lawyers of tomorrow should focus on cooperating with AI, making sure it is developed and used legally, so as to augment their services rather than fearing being replaced by it. The future partner of law firms may be the leader of a multi-disciplinary team of professionals in which AI is substantially used. Legal teams consisting of people and AI could be a dynamic and very effective structure where humans have an important role to play thanks to their unique features and abilities: intellective judgment, empathy, creativity and adaptability.

Keywords:

LawTech, legal technology, future, lawyers of the future, artificial intelligence, AI, algorithms, machine learning, blockchain, technology, legal analytics, transparency, discrimination, judges, law firm, court. 


\section{Introduction}

The revolutionary changes within the legal industry due to new technologies date back to the 1990 s with the arrival of the Internet. With it, the possibility of faster communication via e-mail arrived, the creation of digital libraries, the primordial document management systems and early contract lifecycle management platforms which started to be used by lawyers to facilitate their daily activities.

In recent years there has seen a surge in innovation and investment in legal tech solutions. Lawyers have started using more key technologies such as electronic payments, client portals, and client intake and CRM products ${ }^{1}$. This is because exploiting the advantages of legal tech solutions enables them to be more efficient and productive, more in control of their businesses and acknowledge better the legal needs of clients.

The COVID-19 pandemic had brought about an unprecedented and accelerated adoption of technology by lawyers. The Clio LTR $2020^{2}$ report indicates that the vast majority $(82 \%)$ of law firms are using software to manage their practice, $79 \%$ of lawyers rely on cloud technology to store their firm's data, $62 \%$ of firms allow clients to securely share and sign documents electronically, $73 \%$ allow clients to pay invoices electronically, and $83 \%$ of firms are conducting meetings with clients through remote video conferencing applications.

Remote hearings are nothing new to civil proceedings in some countries like UK (particularly in commercial cases where an international element/party is involved), but the COVID-19 pandemic has forced courts and tribunals in all areas of practice to proceed rapidly to another level, pushing judges, counsels, and participants out of their normal comfort zone. There were three major elements that had caused difficulties to arise: firstly, the lockdown had made the move towards 'full' remote hearings where all participants (including the judge, judge's clerk, counsel, witnesses etc.) are all appearing remotely from separate locations; secondly the speed at which this transition had to take place, and finally, the use of remote hearing in practice areas where remote hearings were uncommon. The use of technology to conduct court hearings had become an inevitable requirement to keep access to justice open and to reduce the backlogs

1 Clio, 'Legal trends for solo law firms' (Clio.com, 2021), <https://www.clio.com/res ources/legal-trends/2021-solo-report/?utm_source=press\&utm_medium=web\&utm _campaign=solo-ltr-2021>, accessed: 13 April 2021.

2 Clio, 'Legal Trends Report' (Clio.com,2020) <https:/www.clio.com/wp-content/up loads/2020/08/2020-Legal-Trends-Report.pdf> accessed: 13 April 2021. 
and delays created by adjournments. The same can be said also for those countries where the use of such technology in the judiciary system has so far been viewed more sceptically (such as in Poland).

The legal profession has undergone fast technological changes but there is so much more that technology can do for lawyers to improve their daily activities, businesses and serve their clients' needs and expectations. The trends indicate that lawyers will be expected to embrace technology even further as technology develops in using LawTech tools. Lawyers will be required to develop their know-how in the use of the underlying technology, and have more advanced digital and computational competences. There is clearly economic pressure for ever-greater use of artificial intelligence (AI) in both low-end and high-end consumers of legal services. The last decade or so has seen a dramatic increase in the capabilities of AI based systems and their application has potential to bring about significant change in the legal sector.

The future for lawyers will substantially be impacted with the use of LawTech tools: it will not only affect the automation of activities and the possibility of replacing certain tasks (e.g. performing routine and repetitive activities or recommendation systems) but it will also see the use of advanced autonomous systems that can cooperate with, and augment certain tasks to deliver greater efficiencies, improve access to know-how and provide better services.

\section{New Means of Production}

The legal ecosystem has evolved through trends taking hold within the industry, and they include data exploitation to enable effective project management, new LawTech products, use of blockchain solutions and AI technologies for the simplification of legal works. These new means of productions have become key drivers of quality and investment in legal technology with in-house legal teams, in law firms, in government legal departments.

A survey from Gartner presented that the proportion of budgets spent by legal departments on technology is set to increase threefold by 2025 , as legal tech solutions have driven lawyers' appetite to expand their use of technology to support workflows and meet productivity demands ${ }^{3}$. Lawy-

$3<$ https://www.gartner.com/smarterwithgartner/5-legal-technology-trends-changingin-house-legal-departments> The Gartner's survey states that legal departments will 
ers are keener to exploit technology primarily to drive efficiency by using it for deal management tools, intellectual property portfolio management, eDiscovery, M\&A due diligence procedures, contract negotiation, data collection and dashboard building, compliance reviews, legal research and more.

Another important area that lawyers will need to learn to exploit is with data. IBM had calculated in 2016 that $90 \%$ of all world's stored data has been created in the past two years ${ }^{4}$, creating new challenges and opportunities for analysis. Data relevant to legal work is no longer just within the sole ambit of accredited legal professionals and is becoming accessible, searchable and analysable to non-lawyers, which means wider competition. Data shows patterns that reveal recurring problems and inefficiencies, and shows insights into how the work is being managed, divided within the office's resources and with which costs. Understanding the value of data will help lawyers rethink who does their work and learn significant information about how to offer better value to their clients and where to look for quality improvement. AI is an invaluable aid in the processing of these huge amounts of data, in searching for hidden correlations that elude the human eye, and in choosing the best solutions.

Blockchain and distributed ledger technology (DLT) are another set of technology that can be used by lawyers to improve their services ${ }^{5}$. DLT can help the legal services become more accessible, transparent, automated and cost efficient. Law firms are leveraging DLT to streamline and simplify their transactional work, to be able to digitally sign documents and to store legal agreements in an immutable way.

For instance, DLT creates a shared ledger accessible by all parties to an agreement, it contains coded-in compliance obligations (through smart contracts, automated contractual terms run by code, embedded in the chain), removing the risk for non-compliance and leaving less room for misinterpretation. Another advantage is the ability to record events for

have automated $50 \%$ of their legal work related to major corporate transactions by 2024, accessed 13 April 2021.

$4<$ https://www.ibm.com/blogs/watson/2016/05/biggest-data-challenges-might-not-ev en-know>, accessed 13 April 2021.

5 In brief blockchain is a type of distributed ledger technology (DLT) that allows for peer-to-peer transactions without the need for a trusted authority (if a public blockchain), creating an immediate, immutable, transparent record of the transaction. They are effective because the transaction is based on a distributed consensus between all nodes on the chain. 
a long period of time, without the possibility for them to be modified without trace or losing any of the judicial authority.

There is a wide range of areas where blockchain could become a major player, from supporting the changing nature of legal work to enabling new lines of business and differentiating service offerings. Practical examples of DLT uses in the legal industry include the recording of music works to protect copyright ${ }^{6}$, safe storage of real estate deeds transforming documents into immutable tokenised assets ${ }^{7}$, time stamp certification of agreements with verifiable digital signature, automation of payments (e.g., management of escrow accounts at a fraction of the cost of manual labour through smart contract $)^{8}$. In short, the transparent, immutable and secure nature of DLT will allow lawyers to solve various types of legal matters, streamline and simplify their transactional work, digitally sign and immutably store legal agreements. Further DLT can also provide more transparency as the shared ledger is accessible by relevant parties, and any such contracts can embed regulatory and/or compliance information, reducing the risk for misinterpretation and/or non-compliance issues. Apparently, lawyers can spend up to $48 \%$ of their time on administrative tasks, including information between software or updating client trust ledgers ${ }^{9}$ which can be significantly eliminated with the use of DLT.

In 2017, PwC revealed that $70 \%$ of surveyed law firms would be open to utilise smart contracts for transactional legal services ${ }^{10}$. What is known, however, is that today there is still a current scepticism around DLT and

6 Silvia A. Carretta, Blockchain challenges to copyright: Revamping the online music industry (Stockholm University 2019) < http://urn.kb.se/resolve?urn=urn:nbn:se:su: diva-173248> accessed 13 April 2021.

7 Shelter Zoom, based in New York, is the first real estate technology startup to incorporate blockchain technology within the real estate industry's infrastructure using Docuwalk, a blockchain-based platform that transforms documents and contracts into immutable, interoperable tokenized assets on the Blockchain.

8 Neel Kirit and Priya Sarkar, 'EscrowChain: Leveraging Ethereum Blockchain as Escrow in Real Estate', (2017) 5, 10 International Journal of Innovative Research in Computer and Communication Engineering, <https://www.researchgate.net/p ublication/325392683_EscrowChain_Leveraging_Ethereum_Blockchain_as_Escro w_in_Real_Estate> accessed 21 June 2021.

9 < https://www.clio.com/resources/legal-trends/2018-report $>$ accessed 20 April 2021.

10 PwC, 'Time for change PwC Law Firms' Survey 2017' (pwc.fr, 2017) <https://ww w.pwc.fr/fr/assets/files/pdf/2017/12/law-firms-survey-report-2017.pdf > accessed 13 April 2021. Out of the analysis of total legal firms interviewed, $41 \%$ will use blockchain for transactional legal services, $21 \%$ for business support and $31 \%$ for providing high-value legal services. 
blockchain which now holds little practical appeal for lawyers due to the lack of a minimal viable ecosystem. Until such an ecosystem is created law firms and in-house legal departments will not be able to take full advantage of this technology.

Lawyers could obtain many advantages by embracing the latest technologies involving artificial intelligence (AI). In the last five years there has been a hype around the implementation of AI in every industry and the legal industry is no exception. AI is posed to develop fast and to bring together an incremental change for lawyers that are willing to increasingly embrace these new opportunities offered by AI to jump-start automation, efficiency, and interconnectivity of its operations.

For example, legal AI tools have been developed to help lawyers review a large number of documents in instances of M\&A procedures. ${ }^{11}$ But that is not just it: the speed at which AI algorithms are developing, becoming better and faster at learning, promises a fast journey into the reality of natural language processing tools for leveraging legal text data, to extract more quickly and precisely information from a huge data set of legal documents (saving lots of hours of manual labour). Through both machine learning and deep learning, AI systems have the ability to mimic, to some extent, human decision-making. These AI tools could be used for text summaries, extracting attributes and relations, document relevance scoring, predicting outcomes (e.g. likelihood of certain crimes being committed in the future or to infer statistical commonalities between judgements to decide how to proceed on a case) and also for answering legal questions. ${ }^{12}$

In commercial transactions, $\mathrm{AI}$ is employed to scan voluminous documentation to identify unusual, unexpected, or disadvantageous contractual clauses ${ }^{13}$. In a recent study, experienced US corporate lawyers were pitted against the LawGeex AI to spot issues in non-disclosure agreements. The

11 Philip Hacker, Ralf Krestel and Sstefan Grundmann and others, 'Explainable AI under contract and tort law: legal incentives and technical challenges' (2020) 28 Artificial Intelligence Law 415-439 <https://doi.org/10.1007/s10506-020-09260-6> accessed 20 April 2021.

12 John Nay, 'Natural Language Processing and Machine Learning for Law and Policy Texts' (7 April 2018), <https://ssrn.com/abstract=3438276> accessed 21 June 2021.

13 Joe Dysart, 'AI Removes the Drudgery from Legal Due Diligence' (Communications of the ACM, 8 January 2019) <https://cacm.acm.org/news/233886-ai-rem oves-the-drudgery-from-legal-due-diligence/fulltext $>$ accessed 19 April 2021; Lauri Donahue, 'A Primer on Using Artificial Intelligence in the Legal Profession' (JOLT Digest, 3 January 2018) <https://jolt.law.harvard.edu/digest/a-primer-o n-using-artificial-intelligence-in-the-legal-profession> accessed19 April 2021; 
lawyers achieved an $85 \%$ success rate, against the Al's $94 \%$; lawyers took 92 minutes on average to complete the task which took the AI 26 seconds ${ }^{14}$. Advances in AI have encouraged non-lawyers in the US to offer services that were otherwise the exclusive domain of lawyers. Several US states have begun to consider licensing non-lawyers to provide some limited legal services: a practice known as "Limited-License Legal Technicians" $(\text { LLLTs })^{15}$.

It is noteworthy that e-disclosure in common law litigation has advanced significantly since the simple keyword search method. Now algorithms are used efficiently for predictive coding, where the capacity of the AI system is employed to learn to respond to unprogrammed situations by the lawyer. This process has been endorsed by the English High Court ${ }^{16}$ as appropriate to consider in reducing costs and increasing efficiency, avoiding necessary delays to the effective resolution of cases. In the US it has been claimed that AI discovery has a better track record than human review ${ }^{17}$.

The CaseCruncher Alpha program ${ }^{18}$ took a challenge between 100 lawyers and the CaseCruncherAI system in predicting the outcome of 775 Payment Protection Insurance Claims likely to be made by the Financial Ombudsman. The CaseCruncher AI System won not only in speed but with an accuracy rate of $86.6 \%$ compared with $66.3 \%$ by the lawyers. AI systems have been trained to predict the outcome of court proceedings ${ }^{19}$. Im-

Chris Goodman, 'AI/Esq: Impacts of Artificial Intelligence in Lawyer-Client Relationships' (2019) 72 Okla. L. Rev. 149.

14 LawGeex, 'Comparing the Performance of Artificial Intelligence to Human Lawyers in the Review of Standard Business Contracts' (LawGeex, 2018), <http://ai.law geex.com/rs/345-WGV-842/images/LawGeex\%20eBook\%20Al\%20vs\%20Lawyers \%202018.pdf $>$ accessed 20 April 2021.

15 B. Sheppard, 'Incomplete Innovation and the Premature Disruption of Legal Services' (2015) 1797 Mich. St. L. Rev. 1842. A State of California task force called for the bar to consider a pilot programme for LLLTs: <http://board.calbar.ca.gov/ docs/agendaItem/Public/agendaitem1000013042.pdf > accessed 19 April 2021.

16 Pyrrho Investments Ltd $v$ MWB Property Ltd [2016] EWHC 256 (Ch) and David Brown - $v$ - BCA Trading Ltd \& Others [2016] EWHC 1464 (Ch). The learning strategy of predictive coding is described in: Richard Bolton and David Hand, 'Unsupervised profiling methods for fraud detection' (2001) Proceedings of credit scoring and credit control VII , 235-255.

17 Federal Housing Finance Agency $v$ HSBC North America Holdings Inc, Nos 11 Civ. 6189 (DLC), 11 Civ, 2014 WL 1909446, at 1 (S.D.N.Y. May 13, 2014).

$18<$ https://www.case-crunch.com/\#challenge> accessed 20 April 2021.

19 Reed C. Lawlor, 'What computers can do: analysis and prediction of judicial decisions' (1963) 49 American Bar Association Journal 337 cited in: Nikolaos 
pressive results have been achieved in predicting decisions of the European Court of Human Rights ${ }^{20}$, and of the US Supreme Court ${ }^{21}$. Such statistical analysis is sometimes used by law firms to decide whether to take on cases $^{22}$. However, it is questionable how accurate these AI systems will play when it comes to assessing the exercise of judicial discretion or dealing with novel or complicated cases. Machine Learning systems are developing legal research skills by employing user-feedback to train algorithms to move beyond keyword searches and perform ever more demanding tasks ${ }^{23}$.

\section{Hybrid Disruption Through Lawtech}

We are in the middle of a 'hybrid disruption' in terms of implementation of new technologies within the legal industry. LawTech empowers lawyers in better doing their work, reducing repetitive works, allowing them to focus more on their specialisations, and bringing better value to their clients. Brian Zubert, Director of Ecosystem Development of Thomson Reuters, when asked about the past and present of the legal profession, stated that:

"Investment in Legal Tech has grown substantially in recent years, and so too have the number of solutions and services available. Although law firms and corporate legal departments have more choices than ever, the reality is that there are more options available than purchasing budget, procurement capacity, integration capability, rollout management, and investment

Aletras and others , 'Predicting Judicial Decisions of the European Court of Human Rights: A Natural Language Processing Perspective’ (2016) Peer J. Comput. Sci. $<$ https://peerj.com/articles/cs-93.pdf $>$ accessed 19 April 2021.

20 Aletras and others, (n 22).

21 Matthew Hutson, 'Artificial Intelligence Prevails at Predicting Supreme Court Decisions', (Science, 2 May 2017), <https:/www.sciencemag.org/news/2017/05/art ificial-intelligence-prevails-predicting-supreme-court-decisions $>$ accessed 19 April 2021.

22 Deloitte, 'Objections Overruled: The Case for Disruptive Technology in the Legal Profession' (Deloitte, 2017), available $<$ https:/www2.deloitte.com/content/dam/D eloitte/uk/Documents/corporate-finance/deloitte-uk-technology-in-law-firms.pdf accessed 19 April 2021.

23 Brian Sheppard, 'Does Machine-Learning-Powered Software Make Good Research Decisions? Lawyers Can't Know for Sure' (Legal Rebels, 22 November 2016) <http:/www.abajournal.com/legalrebels/article/does_machine-learning-po wered_software_make_good_research_decisions_lawyers> accessed 19 April 2021. 
needed to drive meaningful user adoption. 2020 magnified the operating constraints and competing for technical priorities"24.

While automation may substitute some tasks for lawyers, the ability to undertake other tasks augmented by AI technologies becomes more valuable ${ }^{25}$. As AI acquires more advanced skills it may reach a stage where it would be seen as an alternative to many lawyers' tasks ${ }^{26}$. We suggest that: (a) some legal tasks will remain beyond the capabilities of AI for the foreseeable future and continue to be performed by lawyers; (b) some tasks (predominantly the repetitive and/or administrative) will be substituted by AI systems; and (c) new tasks will be created in order to be delivered through AI systems legitimately, which will be carried out by multidisciplinary teams consisting of lawyers and other experts working together.

Professor Brian Sheppard outlined the possible effects of Al's "disruptive innovation" on legal services ${ }^{27}$; in his view AI will not entirely replace lawyers in the foreseeable future, but it could make sufficient inroads to disrupt the economics of the legal profession; a process which he refers to as 'premature disruption, since it would provide only some, but not all, legal services. Premature disruption would affect the profitability of the legal profession without offering an alternative to the core services that lawyers offer. Core services consist of the creative intellectual tasks of the lawyer like arguing difficult cases, advancing novel legal interpretations, developing legal concepts and generally advancing the study of the law.

AI could reduce demand for lawyers' core services or lead to a fall in their profitability or bring down the number of lawyers needed to provide such services ${ }^{28}$. Demand for core lawyer services could diminish because

24 Kenneth Jones and Matthew Jones, 'Strategies supporting the development and deployment of high-quality legal software 221)' (Legal evolution blog, 31 January 2021) < https://www.legalevolution.org/2021/01/tactics-supporting-the-developme nt-and-deployment-of-high-quality-legal-software-221> accessed 20 April 2021.

25 Andrew McAfee and Erik Brynjolfsson, 'Human Work in the Robotic Future: Policy for the Age of Automation' (2016) 95, 4 Foreign Affairs 139-150<https://w ww.jstor.org/stable/43946940> accessed 21 June 2021.

26 John O. McGinnis and Russell G. Pearce, 'The Great Disruption: How Machine Intelligence Will Transform the Role of Lawyers in the Delivery of Legal Services' (2013) 82 Fordham L. Rev. 3041.

27 Sheppard (n 18). See generally, Clayton Christensen, 'Disruptive Innovation' (2020), <http://www.claytonchristensen.com/key-concepts> accessed 20 April 2021.

28 It seems that this process is already underway with large firms restricting recruitment: The Law Society, 'Law Society report, Legal services sector forecasts 20172025’ ( Law Society of England and Wales, 2018) <https:/www.lawsociety.org.uk 
clients may be content with the limited but cheaper services offered by AI. For example, instead of litigating, parties may settle in accordance with AI-approximated predictions of the outcome of litigation. The profitability of litigation services themselves may fall as profits from peripheral activities decline due to automation; because, for instance, disclosure and document review have been fully automated, or mediation is conducted by machines. ${ }^{29}$

However, the development and implementation of AI systems in LawTech to perform tasks (whether they be substituted or augmented tasks) will require human capital and intelligence for it to be legitimately functional so as to have sufficient trust and confidence in the AI system. This will create new roles for lawyers working in multidisciplinary teams with other professionals, not excluding AI itself (i.e. centaurs AI) ${ }^{30}$.

Further, the use of LawTech and AI in society generally will bring about novel legal issues and challenges, for example in the way intellectual property laws may have to be adapted and applied. These will need more intellectual input from lawyers to steer proper evolution and governance of laws.

/support-services/research-trends/legal-services-sector-forecasts $>$ / accessed 20 April 2021.; Jane Croft, 'More than 100,000 legal roles to become automated' (Financial Times, 15 March 2016), <https://www.ft.com/content/c8ef3f62-ea9c-11e5-888e-2e add5fbc4a4>, accessed 20 April 2021. Consultancy firm McKinsey estimates that $22 \%$ of a lawyer's job and 35\% of a paralegal's job can be automated: Michael Chui, James Manyika and Mehdi Miremadi, 'Four fundamentals of workplace automation' (McKinsey Digital, 1 November 2015) <https:/www.mckinsey.com/ business-functions/mckinsey-digital/our-insights/four-fundamentals-of-workplace -automation> accessed 20 April 2021.

29 E.g. mediation: University of Southern California, 'Do we trust artificial intelligence agents to mediate conflict? Not entirely' (Science Daily, 16 October 2019) $<$ https://www.sciencedaily.com/releases/2019/10/191016094909.htm> accessed 21 June 2021.

30 Nicky Case, 'How To Become A Centaur' (Journal of Design and Science MIT Media Lab, 02 February 2018), <https://jods.mitpress.mit.edu/pub/issue3-case/rel ease/6> accessed 12 April 2021.; Hilary G. Escajeda, 'The Vitruvian Lawyer: How to Thrive in an Era of AI and Quantum Technologies' (2020) XXIX Kansas J. of Law \& Pub. Pol'y 421-521 , 463), <https://ssrn.com/abstract=3534683> accessed 14 March 2021. 


\section{New Challenges Posed by the Use of AI}

While the benefits of AI are clear - e.g. cost-effective legal services, faster outcomes, greater consistency - there are also novel challenges and risks that have to be considered by lawyers.

Dr. Paola Cecchi-Dimeglio, a behavioural scientist and senior research fellow for Harvard Law School's Centre on the Legal Profession, stated in an interview that it's very important for legal organisations or companies in general to determine why they are using AI in the first place: "You have to remember that with many legal organisations, the data they are looking at is either what is publicly available or data they have gathered from working with their clients. And when artificial intelligence starts working with this data, it can be a very positive thing for a law firm" ${ }^{31}$. She noted that this process allows firms to make better decisions about jurisdictions, judges' decisions, and client matters in comparable situations. But wisely she added that "problems arise, especially problems with biases, when the organization isn't careful about where it's taking its data from or about what portion of data it's using and not using. Because if you start out with a biased history, you're going to have biased results." 32

A classic example of how incompetency in the use of technology can cause substantial injustice is illustrated in the case of the COMPAS algorithm, ${ }^{33}$ which was used by US judges for assessing the probability of reoffending. The algorithm was based on questions which indirectly discriminated against black humans, who were given relatively disproportionate sentences in comparison to white humans. Algorithm bias can be a troublesome problem which has attracted considerable criticism. ${ }^{34}$

31 Thomson Reuters Institute, Ask Dr. Paola: Detecting \& Battling Biases in Artificial Intelligence \& Machine Learning (Part 2), March 29, 2018. < https://www.legalexecut iveinstitute.com/ask-dr-paola-battling-ai-biases-march>accessed 13 April 2021.

32 Thomson Reuters Institute (n. 34).

$33<$ https://www.propublica.org/article/machine-bias-risk-assessments-in-criminal-sen tencing $>$, accessed 29 April 2021.

34 Law Society Commission on the Use of Algorithms in the Justice System, Algorithms in the Criminal Justice System (2019) < https://www.lawsociety.org.uk/top ics/research/algorithm-use-in-the-criminal-justice-system-report $>$ accessed 23 September 2021. Liberty have called for a ban on the use of such algorithms. See: I. Iberty, 'Liberty Report Exposes Police Forces' Use of Discriminatory Data to Predict Crime'(libertyhumanrights.org.uk, 4 February 2019), <https://www.libert yhumanrights.org.uk/issue/liberty-report-exposes-police-forces-use-of-discriminato ry-data-to-predict-crime > accessed 20 April 2021. 
The main challenges to be address by the use of AI systems include:

Bias and discrimination: the data sample used to train and test the AI system can often be insufficiently representative of the populations from which they are drawing inferences. This creates real possibilities of biased and discriminatory outcomes, because the data being fed into the systems is flawed from the start. Secondly, it should be noted that as AI technologies gain their insights from the existing structures and dynamics of the societies they analyse, so data-driven technologies can reproduce, reinforce, and amplify the patterns of marginalisation, inequality, and discrimination that exist in these societies. Further, because many of the features, metrics, and analytic structures of the models that enable data mining are chosen by their designers, these technologies can potentially replicate their designers' preconceptions and biases.

Lack of transparency, black box effect: transparency of legal decision-making is a requirement of fairness and accountability, it bolsters public confidence and promotes legitimacy ${ }^{35}$. Many machine learning models generate their results by operating on high dimensional correlations that are beyond the interpretive capabilities of human scale reasoning. In these cases, the rationale of algorithmically produced outcomes that directly affect decision subjects remains opaque to those subjects. While in some use cases, this lack of explainability may be acceptable, in some applications, where the processed data could harbour traces of discrimination, bias, inequity, or unfairness, the opaqueness of the model may be deeply problematic. Quite apart from considerations of fairness and accountability, lack of transparency may conceal overestimation of the reliability of AI algorithms' outcomes. Facial-recognition results, for instance, could be taken as conclusive, overlooking the fact that the system produces false positives in 20 to $34 \%$ of $\operatorname{cases}^{36}$. Solutions to the

35 More on AI Transparency: Gabriela Bar, Explainability as a legal requirement for Artificial Intelligence, (Medium.com, November 2020) $<$ https://medium.com/wom eninai/explainability-as-a-legal-requirement-for-artificial-intelligence-systems-66da 5a0aa693>, accessed: 12/04/2021.

36 Alice Feng and Shuyan Wu, 'The myth of the impartial machine' (Parametric Press, 1 May 2019) <https://parametric.press/issue-01/the-myth-of-the-imparti al-machine $>$. Margot Kaminski, 'Binary Governance: Lessons from the GDPR's approach to Algorithmic Accountability' (2019) 92 S. Cal. L. Rev. 1529); Monika Zalnieriute, Bennett Moses and George Williams, 'The Rule of Law and Automation of Government Decision-Making' (2019) 82 M.L.R. 425. European Parliamentary Research Service, 'A governance framework for algorithmic accountabi- 
explainability of AI algorithms have been considered with the use of counterfactuals which identify the prime factors that lead to the outcome, in order to identify and neutralise any unfairness. ${ }^{37}$ It is also to be discussed whether it would be easier to deal with algorithm bias than with human unconscious bias ${ }^{38}$.

Privacy and Data Protection: with the use of big data, protecting privacy, client confidentiality, and legal professional privilege have to be carefully considered when designing, and using AI systems.

Isolation and Disintegration of Social Connection: Excessive automation might reduce the need for human-to-human interaction limiting exposure to worldviews and peer coordination and might polarise relationships and views. This may reduce the development of emotional interactive skills for future lawyers as well as the quality of client relationships.

Lawyers will have to be constantly aware of the challenges and ethical concerns on the uses of $\mathrm{AI}$ in both the legal sector and in any client sector specific technology used, (e.g., FinTech, InsurTech) as well as the growing laws and regulations surrounding the use and deployment of the technology. It is particularly important in the context of compliance with the principles of professional ethics and professional secrecy. An interesting idea may be a system of conformity assessment (digital certification) for lawyers using advanced AI systems to provide legal services.

Although the full adaptation of AI is still in its infancy in many areas of the law, as mentioned above there are already issues around the use of AI that have raised to the surface with regard to ethics, data protection, fundamental rights, discrimination and bias passed down from humans to the AI. Like many similarly situated industries across world markets, it may be wise for the legal industry to pause before jumping blindfolded on

lity and transparency', Panel for the Future of Science and Technology (STOA 2019)), p.64, <http://www.europarl.europa.eu/stoa/en/document/EPRS_STU(2019 624262> accessed 20 April 2021.

37 Sandra Watcher, Brent Mittelstadt and Chris Russel, 'Counterfactual explanations without opening the black box: automated decisions and the GDPR' (2018) 31, 2 Harvard Journal of Law and Technology $<$ https://jolt.law.harvard.edu/assets/artic lePDFs/v31/Counterfactual-Explanations-without-Opening-the-Black-Box-Sandra -Wachter-et-al.pdf> accessed 20 April 2021.

38 Holger Spamann and Lars Klöhn, 'Justice is Less Blind, and Less Legalistic, Than We Thought: Evidence from an Experiment with Real Judges' (2017) 45 J.L.S. 255. They found that judges' decisions were affected by irrelevant characteristics of defendants but that they had failed to mention these in their judgments. 
using a new technology that is not fully understood. The implementation of AI innovative solutions in the legal industry has really only just started. We need to see how it will play out over the next decade or two, to see whether AI will truly bring a disruptive transformation to the legal sector.

\section{Innovation as a Must on the Long Run}

LawTech solutions are a key part of the disruption and development within the legal industry. But technology alone cannot be the sole disruptive factor. Lawyers are expected to integrate the use of technology with specialised knowledge with regard to how each new technology works. The lack of training in tech innovation and related disciplines makes it challenging for lawyers to be able to make conscious and long-lasting decisions to their business models and be successful in the long run. Lawyers will have to master the basics of legal technologies available on the market and this requires enormous talent, energy, commitment and skill.

This is also the approach supported by bar associations, which understand the need for their members to become more knowledgeable in terms of new technologies. For instance, the American Bar Association changed its rule on lawyer competency to requiring that lawyers have duty to "keep abreast of changes in the law and its practice, including the benefits and risks associated with relevant technology" 39 to maintain the requisite knowledge and skill. The Law Society and the Bar Council of England and Wales takes a continuing view to keep members informed of relevant guidance and training, and work with relevant authorities to deal with novel use of technologies.

The next few years will require from lawyers a rethinking of the legal profession as we know it. It will be necessary to fully understand how the changed picture of our post-2020 society may have also changed the nature of the legal profession (not only from a technological perspective) with new services that must replace traditional ones and new market areas that are emerging on the horizon. Alongside, there are the issues of the necessary digitalisation of large spaces of the legal profession and the automation of various routine processes. In addition to the vexed

39 American Bar Association, 'Model Rules of Professional, Rule 1.1 - Competence, Comment' (American Bar Association, August 2020) <https://www.americanbar.o $\mathrm{rg}$ /groups/professional_responsibility/publications/model_rules_of_professional_ conduct/rule_1_1_competence/comment_on_rule_1_1> accessed 20 April 2021. 
question of liberalisation, due to the emergency of a saturated market and high competition, it will be necessary to rethink how lawyers offer their services virtually, modernising their billing and payment structures, including price transparency and flexibility, unbundling legal services offline, and offering specialised services.

At the end of the day, meeting the needs of firms, legal departments, technology companies, vendors, and so on, requires a team effort in pursuit of a joint ambition: getting legal work done simply, efficiently, and accurately. This is a great first step, but it must be substantiated with action and engagements discussion in detail. Until lawyers are willing to invest in gaining legal tech knowledge, knowhow and solutions, there will be a large delay in the development for LawTech solutions in the legal industry.

The future direction of LawTech development is worth considering now. Even if AI becomes solid enough that we can be sure that the AI applies the right rules, takes all circumstances into account, and is impartial, will we be able to understand the logical argument carried out by the algorithm to trust its decision? Will AI ever be able to consider the thousands shades of grey that human life generates, which have a significant impact on the interpretation of declarations of will and the resolution of litigation?

It seems that the lawyers of tomorrow should focus on using AI to augment their services rather than the fear of being replaced by it. The future partners of the law firm may be leaders of a multi-disciplinary, multi-diverse team of professionals in which AI is substantially implemented and used. Legal teams consisting of people using AI could be a dynamic and very effective structure where humans have an important role to play, thanks to their unique features and abilities: intellective judgment, empathy, creativity and adaptability. 
